\title{
The Front-End and Slow Control boards for the Wide Field of View Cherenkov Telescopes of LHAASO.
}

\section{G. Di Sciascio*, P. Cipollone, C. Di Donato, G. Masciantonio, F. Palma, R. Sparvoli} INFN - Roma Tor Vergata, Italy

E-mail: disciasciodroma2.infn.it

\author{
A. Chiavassa ${ }^{a, b}$, M. Da Rocha Rolo ${ }^{b}$, G. Dellacasa ${ }^{b}$, A. Rivetti ${ }^{b}$, F. Rotondo ${ }^{b}$, P. \\ Vallania $^{b, c}, \mathbf{S}$. Vernetto ${ }^{b, c}$, C. Vigorito ${ }^{a, b}$ \\ ${ }^{a}$ Dipartimento di Fisica dell'Università di Torino, via P. Giuria 1, 10125 Torino, Italy \\ ${ }^{b}$ INFN, Sezione di Torino, via P. Giuria 1, 10125 Torino, Italy \\ ${ }^{c}$ INAF, Osservatorio Astrofisico di Torino, via P. Giuria 1, 10125 Torino, Italy
}

\section{for the LHAASO Collaboration}

The LHAASO (Large High Altitude Air Shower Observatory) experiment is currently under installation at high altitude (4410 m a.s.1., $600 \mathrm{~g} / \mathrm{cm}^{2}$ ) in the Daocheng site, Sichuan province, P.R. China, with the aim of studying with unprecedented sensitivity the spectrum, the composition and the anisotropy of cosmic rays in the energy range between $10^{12}$ and $10^{18} \mathrm{eV}$, as well as to act simultaneously as a wide aperture (about $2 \mathrm{sr}$ ), continuously operating gamma-ray telescope in the energy range between $10^{11}$ and $10^{15} \mathrm{eV}$. One of the main detectors of LHAASO is constituted by an array of 18 wide field of view $\left(16^{\circ} \times 16^{\circ}\right)$ Imaging Atmospheric Cherenkov Telescopes (IACTs). Unlike the usual IACTs dedicated mainly to gamma-ray astronomy, these telescopes will be devoted to the study of the energy spectrum and elemental composition of the cosmic rays around the "knee" of the all-particle primary spectrum (at about $3 \cdot 10^{15} \mathrm{eV}$ ). The focal plane of these telescopes being made of SiPMs, dedicated Front-End (FE) and Slow Control (SLC) boards must be designed with the needed amplification and bandwidth features to achieve the required wide dynamic range of 10-32,000 photoelectrons. In this paper, the electronic scheme and the components selection of these boards are presented and discussed.

35th International Cosmic Ray Conference - ICRC2017

10-20 July, 2017

Bexco, Busan, Korea

\footnotetext{
* Speaker.
} 


\section{The LHAASO experiment}

The Large High Altitude Air Shower Observatory (LHAASO) project is a new generation instrument, to be built at 4410 meters of altitude in the Sichuan province of China, with the aim to study with unprecedented sensitivity the spectrum, the composition and the anisotropy of cosmic rays in the energy range between $10^{12}$ and $10^{18} \mathrm{eV}$, as well as to act simultaneously as a wide aperture (about two sr), continuosly-operated gamma ray telescope in the energy range between $10^{11}$ and $10^{15} \mathrm{eV}$. The remarkable sensitivity of LHAASO in cosmic rays physics and gamma astronomy would play a key-role in the comprehensive general program to explore the High Energy Universe.

The first phase of LHAASO will consist of the following major components (see Fig. 1):

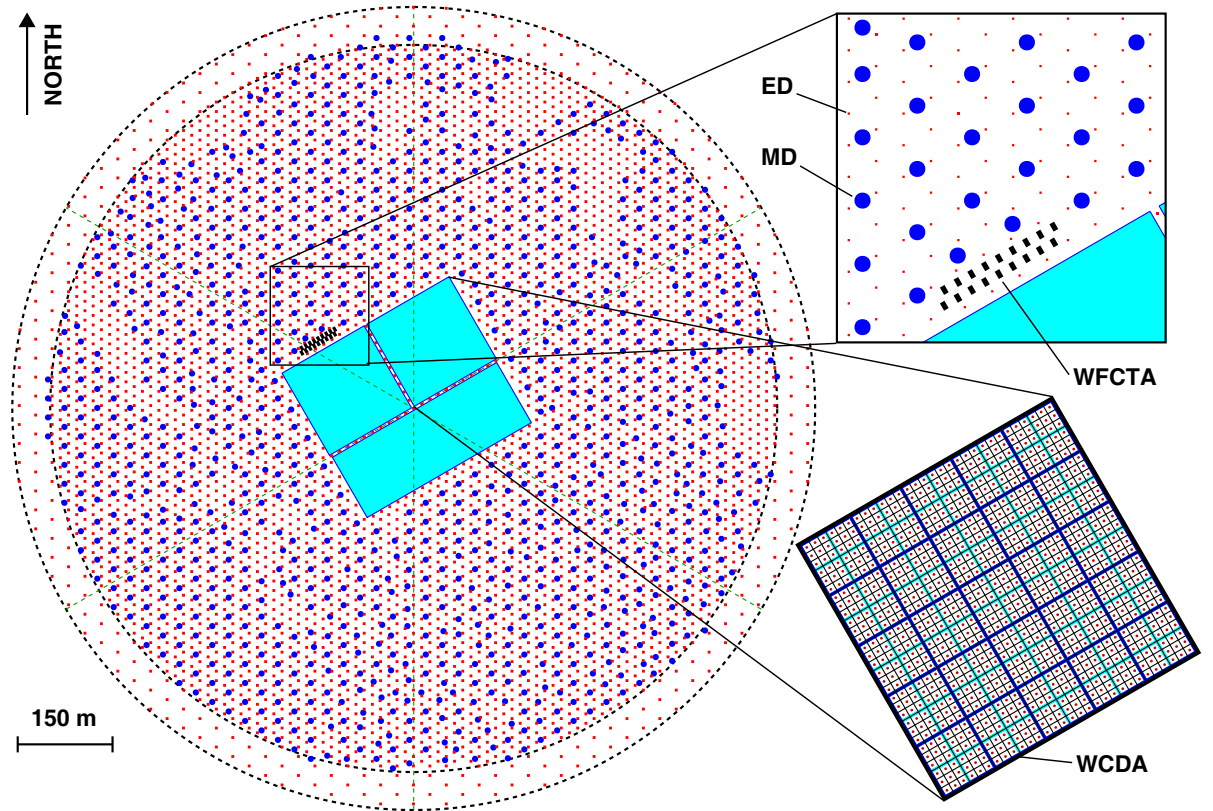

Figure 1: Layout of the LHAASO experiment. The insets show the details of one pond of the WCDA and of the KM2A array constituted by two overimposed arrays of electromagnetic particle detectors (ED) and of muon detectors (MD). The telescopes of the WFCTA, located at the edge of a pond, are also shown.

- $1.3 \mathrm{~km}^{2}$ array (LHAASO-KM2A), including 5195 scintillator detectors $1 \mathrm{~m}^{2}$ each, with 15 $\mathrm{m}$ spacing, for electromagnetic particle detection.

- An overlapping $1 \mathrm{~km}^{2}$ array of 1171 underground water Cherenkov tanks $36 \mathrm{~m}^{2}$ each, with $30 \mathrm{~m}$ spacing, for muon detection (total sensitive area $\sim 42,000 \mathrm{~m}^{2}$ ).

- A close-packed, surface water Cherenkov detector facility with a total area of about 78,000 $\mathrm{m}^{2}$ (LHAASO-WCDA).

- 18 wide field-of-view air Cherenkov (and fluorescence) telescopes (LHAASO-WFCTA).

- An array of neutron monitors to study the hadronic component of showers. 
LHAASO will be located at high altitude (4410 m asl, $600 \mathrm{~g} / \mathrm{cm}^{2}, 29^{\circ} 21^{\prime} 31^{\prime \prime} \mathrm{N}, 100^{\circ} 08^{\prime} 15^{\prime \prime}$ E) in the Daocheng site, Sichuan province, P.R. China. The commissioning of a quarter of the experiment is expected in 2018. The conclusion of installation in 2021.

LHAASO will enable studies in cosmic ray physics and gamma-ray astronomy that are unattainable with the current suite of instruments:

1) LHAASO will perform a sky survey of the Northern sky with a detection threshold of $\sim 0.03$ Crab unit at $\mathrm{TeV}$ energies and $\sim 0.1 \mathrm{Crab}$ around $100 \mathrm{TeV}$ in one year. This unique detector will be capable of continuously surveying the $\gamma$-ray sky for steady and transient sources from $100 \mathrm{GeV}$ to $1 \mathrm{PeV}$.

2) The sub-TeV/TeV LHAASO sensitivity will allow to observe AGN flares that are unobservable by other instruments, including the so-called $\mathrm{TeV}$ orphan flares. Multi-wavelength observations of AGN flares from radio to TeV probe the environment up to within $\sim 0.01 \mathrm{pc}$ of the super-massive black hole constraining models of gamma ray production and acceleration of charged particles.

3) LHAASO will study in detail the high energy tail of the spectra of most of the $\gamma$-ray sources observed at $\mathrm{TeV}$ energies, opening for the first time the $\mathrm{PeV}$ range to the direct observation of the high energy cosmic ray sources. When new wavelength bands are explored in astronomy, previously unknown sources and unknown types of sources are discovered. LHAASO's wide field of view provides therefore a unique discovery potential.

4) LHAASO will map the Galactic diffuse gamma-ray emission above few hundreds $\mathrm{GeV}$ and thereby measure the cosmic ray flux and spectrum throughout the Galaxy with high sensitivity. The measurement of the space distribution of diffuse $\gamma$-rays will allow to trace the location of the CR sources and the distribution of interstellar gas.

5) LHAASO will allow to reconstruct the energy spectra of different mass groups in the $10^{12}$ $10^{18} \mathrm{eV}$ range with unprecedented statistics and resolution, thus tracing the light and heavy components through the knee of the all-particle spectrum.

6) LHAASO will allow to measure, for the first time, the CR anisotropy across the knee separately for light and heavy primary masses.

7) The different observables (electronic, muonic and Cherenkov/fluorescence components) that will be measured in LHAASO with unprecedented resolution will allow a detailed investigation of the role of the hadronic interaction models, therefore investigating if the EAS development is correctly described by the current simulation codes.

8) LHAASO will allow a search for TeV-scale Dark Matter, with unprecedented sensitivity for a DM particle mass above $10 \mathrm{TeV}$, as well as studies of fundamental physics (Lorentz Invariance Violations, Primordial Black holes, Q-balls, ...).

In the next decade CTA-North and LHAASO are expected to be the most sensitive instruments to study Gamma-Ray Astronomy in the Northern hemisphere from about $20 \mathrm{GeV}$ up to PeV. 


\section{The Wide Field of View Cherenkov Telescope Array (WFCTA)}

One of the main component of the LHAASO experiment is constituted by an array of 18 wide field of view $\left(16^{\circ} \times 16^{\circ}\right)$ Imaging Atmospheric Cherenkov Telescopes (IACTs). Unlike the usual IACTs dedicated mainly to gamma-ray astronomy, these telescopes will be devoted to the study of the energy spectrum and elemental composition of the cosmic rays around the "knee" of the all-particle primary spectrum (at about $3 \cdot 10^{15} \mathrm{eV}$ ). These telescopes in a second phase of the experiment will be operated as fluorescence detectors to extend the energy range up to $10^{18} \mathrm{eV}$.

Each telescope has a spherical mirror of $2.3 \times 2.3 \mathrm{~m}^{2}$ with a radius of curvature of $5500 \mathrm{~mm}$. The camera is located at the focal plane, $2720 \mathrm{~mm}$ away from the reflector centre to optimize the spot shape. The focal plane is covered by 1024 pixels of dimensions $1.5 \times 1.5 \mathrm{~cm}^{2}$ (actually a $2 \times$ 2 array of $0.75 \times 0.75 \mathrm{~cm}^{2}$ innovative SiPM units). A Winston cone is placed on the top of each pixel to have a full coverage of the focal plane. The dimensions of each exagonal Winston cone is $25.3 \mathrm{~mm}$ (side to side, corresponding to a field of view of about $0.5^{\circ} \times 0.5^{\circ}$ ). The total field of view is $14^{\circ} \times 16^{\circ}$. The whole system is hosted in a container with an entrance door of $2.3 \times$ $2.3 \mathrm{~m}^{2}$, sealed by a glass window to ensure a dust-proof environment. The telescopes are remotely controlled for both mechanics and electronics, with a minimum amount of moving parts to have a simple and reliable device.

In this configuration, the hybrid observations of WFCTA and KM2A will allow the measurement of the chemical composition of the primay cosmic rays. The WFCTA will provide the $X_{\max }$ location (height of the shower maximum) through the DIST parameter, i.e. the angular distance between the shower arrival direction and the barycenter of the shower image on the focal plane. Around the knee, an iron-generated shower develops faster in the atmosphere, reaching its maximum about $70 \mathrm{~g} / \mathrm{cm}^{2}$ higher than a proton-generated shower with the same total energy, allowing a good separation between the different components. The primary energy is well estimated by the Cherenkov image, that contains a calorimetric information on the shower development in the atmosphere, provided an accurate core location by the KM2A (the expected resolution is $\pm 3 \mathrm{~m}$ ). The muon content, and in particular the ratio between the number of muons and electrons gives a further strong discrimination power. A combination of all these parameters in a multivariate analysis or neural network will allow the composition studies in the knee region.

The capability of the WFCTA to reconstruct showers with high accuracy and the potential of this detector in studying cosmic ray physics is demonstrated by the hybrid measurement of the light $(\mathrm{p}+\mathrm{He})$ component energy spectrum of primary cosmic rays carried out operating one prototype Cherenkov telescope at the Yangbaijing Cosmic Ray Observatory (see Fig. 2) in combination with the ARGO-YBJ experiment [1, 2, 3, 4].

As described in [5], this measurement allowed the observation of the bending of the proton spectrum at about $700 \mathrm{TeV}$.

The telescopes will be rearranged to cover elevations from $3^{\circ}$ to $59^{\circ}$, with 2 additional telescopes covering elevations from $10^{\circ}$ to $24^{\circ}$ from an orthogonal direction. With this configuration we expect that showers above $100 \mathrm{PeV}$ will be detected stereoscopically with a high resolution on $X_{\max }$ measurement. As for the Cherenkov case, the electron and muon content will give further information for chemical composition study and discussion. 


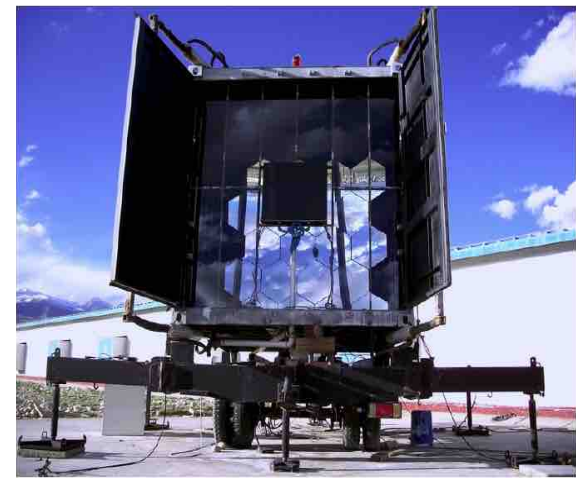

Figure 2: A prototype telescope of the WFCTA.

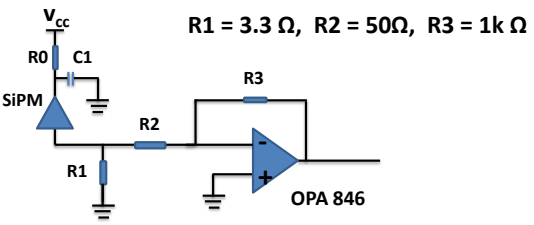

Figure 3: Electronic scheme of the preamplifier circuit for the SiPM output signal.

\section{The Front-End board}

The scheme of a pre-amplifier and shaping circuit in an analogue board depends on the SiPM signal pulse shape. According to the quenching resistor, used to recharge the SiPM after discharging, it can be quite long. In our case, using FBK SiPMs $5 \times 5 \mathrm{~mm}^{2}$, the pulse duration is about 100 ns. Another important effect is due to the total capacitance obtained adding together several SiPMs to obtain the required pixel size of $15 \times 15 \mathrm{~mm}^{2}$. Two different schemes have been tested, the first one based on the AD8012 operational amplifier, and the second one on the OPA846 chip. The optimum performance was obtained with the latter, using the scheme in Fig. 3. The front-end board could then be designed, hosting 16 pre-amplification channels, one for each pixel. The SiPMs are arranged on a mezzanine board, hosting on the other side a temperature sensor (LM94021) to allow the temperature monitoring and a feedback to modify the power supply keeping the gain constant. The SiPM high voltages and the output signals are transferred to the front-end board together with the temperature signal through a connector acting also as a mechanical support of the mezzanine board. The front-end board processes the SiPM output signals and acts as a pass-through to the following Slow Control Board for the temperature output and high voltage. A schematic view of the layout of these boards is shown in Fig. 4

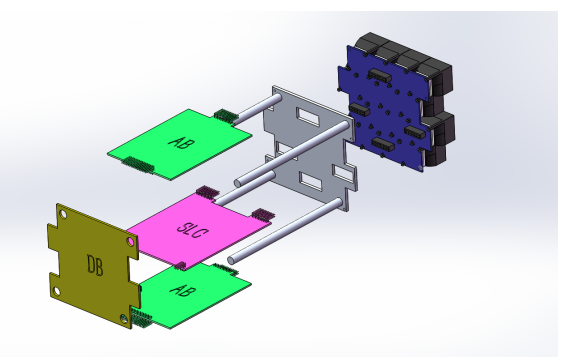

Figure 4: Schematic view of the front-end and back-end boards assembly. The front-end board is the blue one; the others are the analog board (green), the slow control board (pink) and the digital board (ochre).

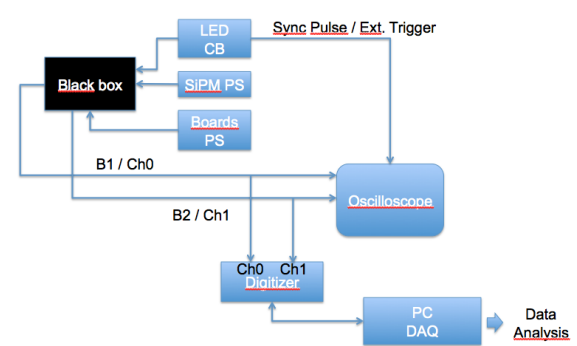

Figure 5: Sketch of the SiPMs and front-end boards test bench. 
The front-end board provides also specifically shaped holes to host on the top of each pixel the Winston Cones, that are fitted into the board. The board has an irregular shape due to the fact that the pixels are not aligned but shifted by half unit on the different raws to reduce the edge effects on the shower images.

To test the boards and their features interfaced to different SiPMs (several producers, SPAD dimensions, quenching resistors, chip dimensions) a Test Bench has been set up. The system sketch is shown in Fig. 5: a black box containing the front-end board (or a test board with one or more preamplifier channels) and the SiPM is lighted up by means of a UV LED placed inside a diffusing sphere. The LED is powered by square waves of different heights, frequencies and duty cycles. The LED lighting can be synchronized to an oscilloscope, and at the same time it is sent to a dual channel digitizer connected to a DAQ PC for data acquisition and analysis. The chosen digitizer is a CAEN DT5751, with up to 4 channels and a bandwidth of 1 Gs/s@ 10 bits, $1 \mathrm{~V}$ range. As for the oscilloscope, the trigger can be authomatic or given by an external signal (the LED lighting). The data analysis includes the peak and charge distributions for the different SiPMs under test. A more complete set of measurements will include:

- I-V curve, to measure the breakdown voltage $\left(V_{b d}\right)$;

- Dark Counts and Cross Talk;

- Gain as a function of $V_{b d}$;

- Gain as a function of temperature;

- $V_{b d}$ as a function of temperature.

This test facility, that will be implemented in Torino, will also be used to check the front-end boards before their integration in the WFCTA camera and following installation at the LHAASO site.

\section{The Slow Control board}

The Slow Control board (SLC) has the task of controlling and distributing the High Voltages (HV) to the SiPMs mounted on the Front-End card. The board allows to adjust 16 independent channels to ensure the gain and the optimal performance for each SiPM.

The check is performed by monitoring the values of temperature and HV applied, and making appropriate corrections to the HV output. The temperature reading is made with a 12 bits ADC, while the reading of the output $\mathrm{HV}$ is made by a 16 bit ADC. The card is also able to implement corrections on $\mathrm{HV}$ with an accuracy of less than $1 \mathrm{mV}$, and provides a selective switch-off of each channel. All the control logic and communication management towards the Digital board (DB), will be implemented on FPGA. To the DB board will be sent the measurements of temperature and $\mathrm{HV}$ for all 16 channels, as well as the corrections applied.

The architecture of the SLC card is made up of 4 main sections (Fig. 6) :

- The temperature monitoring section, 


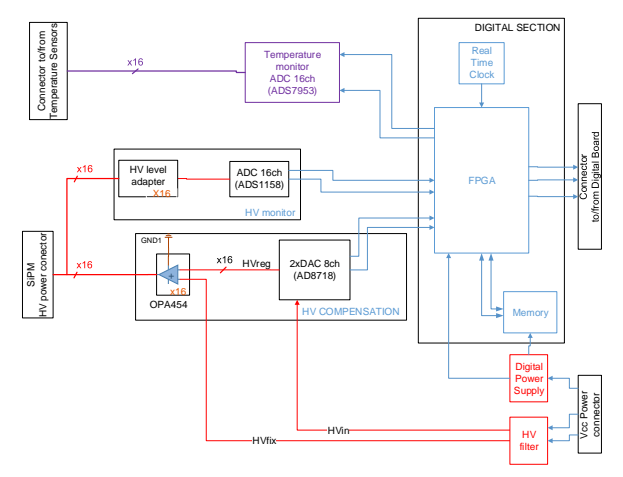

Figure 6: Slow Control board block diagram.

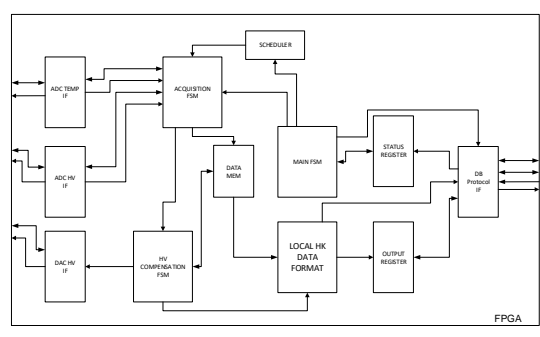

Figure 7: FPGA block diagram.

- The HV compensation section,

- The HV monitoring section

- The digital control section.

The temperature reading section is based on a 16-channel analog to digital converter (ADC). The temperatures are acquired with a resolution of $0.01^{\circ} \mathrm{C}$ and a periodicity of $5 \mathrm{sec}$, and saved within the FPGA, to be used in the algorithm for the HV adjustement. The HV compensation section is based on a group of 16 HV Operational Amplifiers (OPAMP) with power driver features, and two HV Digital to Analog Converters (DACs). The OPAMPs chosen for the SLC card allow to turn off the power of each SiPM, and provide an inner protection for short circuits or load failures. The HV monitoring section is based on a 16ch ADC and an HV level adapter section. The chosen ADC allows to read the HV with a resolution of $0.5 \mathrm{mV}$, the same of the compensation section. The digital section consists of a real time clock (RTC), an FPGA and its memories. The RTC has the task of providing the time reference and to assign a time tag to the corrections made on the HV.

The logic functions are implemented on the FPGA, and can be schematized according to the block diagram in Fig. 7. The Scheduler block has the task of generating the periodic signal to be sent to the state machine that manages the monitoring and acquisition of applied HV values and SiPM temperatures. Interface blocks (ADC TEMP IF, ADC HV IF and DAC HV IF) perform the following functions: control, configuration and management of ADCs and DACs respectively responsible for monitoring functions (HV and temperature) and generation of $\mathrm{HV}$. The acquisition sequence of ADCs is managed by ACQUISITION FSM. The state machine goes into the capture state once the signal generated by the scheduler is received. Once in this state, it sends the startup request to the ADC interface blocks. Once the end acquisition signal is received from the ADC IF, the state machine enters the storage state and saves the data received from the ADCs IF in DATA MEM.

Upon completion of the data storage phase, the ACQUISITION FSM reports to HV COMPENSATION FSM which can start checking the sipm working conditions. The HV COMPENSATION FSM checks whether the HV value applied to each single SiPM is suitable for the correct operation at the readed temperature. In case of non-optimal operating point, this block calculates the new HV to be applied. Once calculated, the data of the new operating point is passed to the 
DAC HV IF, which generates the new HV values. Each time the HV COMPENSATION executes a correction, all corrections are saved to the DATA MEM with a time TAG. All temperature and HV CORRECTIONs information is then packaged and sent to the Digital Board by the LOCAL HK DATA FORMAT block, including:

- Reading / correction time TAG

- SiPM temperature

- HV SiPM before correction

- HV value applied post correction.

. The MAIN FSM block has the task to supervise and coordinate all the functionality of SLC and monitor any SLC working problems. When the SLC is turned on, the main FSM has the important task to schedule the sub blocks power on according to a predetermined sequence, while during normal operations, it has the task of monitoring the alarms of the digital subsections and reporting faults to the DB through the DB PROTOCOL IF block.

Currently a SLC prototype board not including the FPGA has been realized. The digital block is being developed on a Xilinx evaluation board, which can be interconnected by the SLC prototype with appropriate cables. In this way, the debugging of the section containing ADCs and DACs is easier, reducing board production costs in case of errors during the design phase.

\section{Conclusions}

The LHAASO experiment is expected to be the most sensitive detector to study the multi-TeV $\gamma$-ray sky opening for the first time the PeV range to the direct observations of the high energy CR sources. This unique detector will be capable of continuously surveying the $\mathrm{TeV}$ sky for steady and transient sources from $100 \mathrm{GeV}$ to $1 \mathrm{PeV}$.

The installation of an unprecedented muon detection area $\left(\sim 42,000 \mathrm{~m}^{2}\right)$, together with an array of wide field of view Cerenkov/fluorescence telescopes, will allow high resolution selection of showers induced by different primary masses in the range $10^{12}-10^{18} \mathrm{eV}$ and a detailed study of the role of the hadronic interaction models.

\section{References}

[1] J. Liu et al., Astrop. Phys. 67 (2015) 8.

[2] S.S. Zhang et al., Nucl. Instrum. Phys. Res. A629 (2011) 57.

[3] Y.T. Chen et al., Nucl. Instrum. Phys. Res. A795 (2015) 409.

[4] B. Bartoli et al., Chin. Phys. C 38 (2014) 045001.

[5] B. Bartoli et al., Phys. Rev. D 92 (2015) 092005. 